Pacific Journal of Mathematics

SOME PROPERTIES OF THE SORGENFREY LINE AND Re 


\title{
SOME PROPERTIES OF THE SORGENFREY LINE AND RELATED SPACES
}

\author{
Eric K. Van Douwen and Washek F. Pfeffer
}

Any finite power $S^{n}$ of the Sorgenfrey line $S$ has this covering property: if $\varphi(x)$ is a neighborhood of $x$ for each $x \in S^{n}$, then there is a closed discrete subset $D$ of $S^{n}$ such that $\{\varphi(x): x \in D\}$ covers $S^{n}$. No finite power of the Sorgenfrey line is homeomorphic to finite power of the irrational Sorgenfrey line. The Sorgenfrey plane is not the union of countably many nice subspaces.

O. Introduction. All spaces considered are $T_{1}$. The Sorgenfrey line, $S$, is the set of all reals, retopologized by letting all half-open intervals $[a, b)$ be a base. The irrational Sorgenfrey line, $T$, is the subspace of $S$ consisting of all irrational numbers. If $\kappa$ is a cardinal, $S^{\kappa}$ and $T^{\kappa}$ are the product of $\kappa$ copies of $S$ and $T$, respectively. We refer to $S^{2}$ as the Sorgenfrey plane, and to $T^{2}$ as the irrational Sorgenfrey plane.

A neighborhood assignment for a space $X$ is a function $\varphi$ from $X$ to the topology of $X$ such that $x \in \varphi(x)$ for all $x \in X$, [2]. A space $X$ is a $D$-space if for every neighborhood assignment $\varphi$ for $X$ there is a closed discrete subset $D$ of $X$ such that $\{\varphi(x): x \in D\}$ covers $X,[2]$. The space of countable ordinals is not a $D$-space since, as observed in [2]: every countably compact $D$-space is compact. Up to now no satisfactory example of a space which is not a $D$-space is known, where by satisfactory example we mean an example having a covering property at least as strong as metacompactness or subparacompactness.

Since the Sorgenfrey plane is subparacompact, $[8,3.1]$, it is a natural candidate for a satisfactory example of a non- $D$-space. However, we have the following theorem.

THEOREM 1. Every finite power of $S$ is a D-space.

This leaves open the following three questions: Is every finite power of $S$ hereditarily a $D$-space? Is $S^{\omega}$ a $D$-space? Is $S^{\omega}$ hereditarily a $D$-space? A negative answer to any of these questions would be welcome (but is not expected), since $S^{\omega}$ is hereditarily subparacompact, [8, 3.2]. We do not even know if $T^{2}$ is a $D$-space; however, it follows from results in [2] that $S$ is hereditarily a $D$-space. In particular, $T$ is a $D$-space.

The proof of Theorem 1 has suggested a new class of spaces, 
which generalizes the class of left-separated spaces. Recall that a space $X$ is left-separated if there is a well-order $\leqq$ on $X$ such that $\{y \in X: x \leqq y\}$ is open in $X$ for each $x \in X,[6,0.4]$. If $\leqq$ is any reflexive (not necessarily transitive) relation on a set $E$ and $F \subseteq E$, then we shall call $m \in F$ a $\leqq$-minimal element of $F$ whenever $x=m$ for each $x \in F$ with $x \leqq m$.

Definition. A space $X$ is called a generalized left-separated space (abbreviated GLS-space) if there is a reflexive binary relation $\leqq$ on $X$, called GLS-relation, such that

(1) every nonempty closed subset has a $\leqq$-minimal element

(2) $\{y \in X: x \leqq y\}$ is open for each $x \in X$.

THEOREM 2. Every GLS-space is a D-space.

THEOREM 3. Every finite power of $S$ is a GLS-space.

Theorem 1 is an immediate consequence of these two theorems. Other applications of Theorem 2 will be mentioned in $\S 2$. Clearly a subspace of a left-separated space is again a left-separated space. The corresponding statement for GLS-spaces is false.

THEOREM 4. $T$ is not a GLS-space.

In [1] it was shown that $S$ and $T$ are not homeomorphic. Since a closed subspace of a GLS-space is again a GLS-space [see (4.4a)], Theorems 3 and 4 yield the following strengthening of this result.

THEOREM 5. For no positive integers $m$ and $n$ the spaces $S^{m}$ and $T^{n}$ are homeomorphic.

It is apparently unknown if $S^{m}$ and $S^{n}$, or $T^{m}$ and $T^{n}$, can be homeomorphic for distinct positive integers $m$ and $n$.

By a classical result of F. B. Jones, [5], a separable space which has a closed discrete subset of cardinality $c$ is not normal. By a recent result of W. G. Fleissner, [4], such a space is not countably paracompact either. And it is obvious that such a space fails to have many other properties, like metacompactness and collectionwise Hausdorffness. Therefore, the following theorem shows that $S^{2}$, and hence $S^{K}$ for $\kappa \geqq 2$, is not the union of countably many nice subspaces.

THEOREM 6. For every countable family $\mathscr{E}$ of subspaces of $S^{2}$ that covers $S^{2}$ there is an $E \in \mathscr{E}$ which contains a closed separable subspace that has a closed discrete subset of cardinality $c$. 
Since $S^{2}$ is (weakly) $\theta$-refinable, [8, 2.9], it follows that a weakly $\theta$-refinable space need not be the union of countably many metacompact subspaces (the converse is patently true).

The organization of this paper is as follows. Theorem 2 is proved in $\S 1$, where we also consider some of its applications different from Theorem 1. Theorems 3 and 4 are proved in $\S 2$, and Theorem 6 is proved in $\S 3$. In the Appendix we collect some properties of GLS-spaces.

\section{Proof of Theorem 2, and consequences.}

1.1. The proof. Let $X$ be a GLS-space with GLS-relation $\leqq$. Let $\varphi$ be a neighborhood assignment for $X$. Define a new neighborhood assignment $\psi$ for $X$ by

$$
\psi(x)=\{y \in \varphi(x): x \leqq y\} .
$$

It suffices to construct a closed discrete set $D$ in $X$ with $\cup \psi[D]=X$. With transfinite recursion construct, if possible, an $x_{\xi} \in X$ in such a way that for each $x_{\xi}$, when defined,

$x_{\xi}$ is a $\leqq$-minimal element of $A_{\xi}=X-\cup\left\{\psi\left(x_{\eta}\right): \eta<\xi\right\}$.

We can find such an $x_{\xi}$ if $A_{\xi} \neq \varnothing$, since clearly $A_{\xi}$ is closed. Let $\alpha$ be the ordinal at which the construction breaks down because $A_{\alpha}=\varnothing ; \alpha$ exists, for $x_{\xi} \neq x_{\eta}$ if $\xi \neq \eta$. Let $D=\left\{x_{\xi}: \xi<\alpha\right\}$. Then $\cup \psi[D]=X-A_{\alpha}=X$.

It remains to show that $D$ is closed and discrete. To this end it suffices to prove that $\psi(x) \cap D=\{x\}$ for all $x \in D$, since $\cup \psi[D]=X$. Let $x_{\xi} \in \psi\left(x_{\eta}\right)$ for some $\xi, \eta<\alpha$. Clearly $\xi \leqq \eta$, and $x_{\eta} \leqq x_{\xi}$. Both $x_{\eta}$ and $x_{\xi}$ belong to $A_{\xi}$. Consequently $x_{\eta}=x_{\xi}$, as $x_{\xi}$ is $\leqq$-minimal in $A_{\xi}$.

1.2. Consequences. Theorem 2 has been used in [3] for proving that certain spaces are $D$-spaces.

Let $\mathscr{K}(X)$ be the collection of nonempty compact subsets of a Hausdorff space $X$. Equip $\mathscr{K}(X)$ with the so-called Pixley-Roy topology, i.e., basic neighborhoods about $F \in \mathscr{K}(X)$ have the form $\{G \in \mathscr{K}(X): F \subseteq G \leqq U\}$, where $U$ is an open neighborhood of $F$ in $X$. It is noted in [3] that $\mathscr{K}(X)$ is a GLS-space (ordinary inclusion is a GLS-relation). Consequently $\mathscr{K}(X)$ is a $D$-space.

Let $2^{N}$ be the hyperspace of the integers, i.e., the nonempty subsets of $N$, equipped with the Vietoris (or finite, or exponential) topology. It is shown in [3] that $2^{N}$ is a GLS-space (reverse inclusion is a GLS-relation). Consequently $2^{N}$ is a $D$-space. The same 
argument would work for the hyperspace of an uncountable discrete space.

1.3. REMARK. Assume that a GLS-space $X$ admits a GLS-relation satisfying

$(1) \leqq$ is a linear order (or, equivalently, every nonempty closed subset of $X$ has a unique $\leqq$-minimal element);

(2) The intervals $[x, y)=\{z \in X: x \leqq z \prec y\}$ form a base for the topology of $X$.

The technique of the proof in 1.1 can be used to show that $X$ is ultraparacompact. (Recall that a space is ultracompact if every open cover has a disjoint open refinement.) Indeed, let $\mathscr{U}$ be an open cover of $X$. For each $x \in X$, choose $\psi(x)=\left[x, y_{x}\right)$ so that $\psi(x) \subset U$ for some $U \in \mathscr{Q}$. If $D$ is as in 1.1, then $\psi[D]$ is a disjoint open refinement of $\mathscr{U}$.

In the proof of Theorem 3 , we will see that $S$ and $H=S \cap[0, \infty)$ are homeomorphic, and that $\leqq$ is a GLS-relation on $H$. So we have a somewhat unusual proof of the well-known fact that $S$ is ultraparacompact.

2. Proof of Theorems 3 and 4. For the proof of Theorem 3 we will need the following simple lemma.

LEMMA 2.1. Let $\leqq$ be a reflexive and transitive binary relation on a space $X$ such that for every nonempty $\leqq$-chain $K$ in $X$ there is an $m \in K^{-}$with $m \leqq x$ for all $x \in K$. Then each nonempty closed subset of $X$ has $a \leqq-m i n i m a l$ element.

Proof. Let $F$ be a nonempty closed subset of $X$. For every nonempty $\leqq$-chain $K$ in $F$ there is an $m \in K^{-}$with $m \leqq x$ for all $x \in K$; clearly $m \in F$. It follows from the Kuratowski-Zorn lemma that $F$ has a $\leqq$-minimal element.

2.2. Proof of Theorem 3. Let $H$ be $S \cap[0, \infty)$, half the Sorgenfrey line. Then $H$ and $S$ are homeomorphic, for both admit a disjoint open cover by countably many copies of $S \cap[0,1)$. We shall consider $H$ instead of $S$. Let $n$ be nonnegative integer. As usual, the $i$ th coordinate of $x \in H^{n}$ is $x_{i}, 1 \leqq i \leqq n$. Define a reflexive and transitive binary relation $\leqq$ on $H^{n}$ by

$$
x \leqq y \quad \text { if } \quad x_{i} \leqq y_{i} \text { for all } 1 \leqq i \leqq n .
$$

Clearly $\left\{y \in H^{n}: x \leqq y\right\}$ is open in $H^{n}$ for each $x \in H^{n}$. Let $K \cong H^{n}$ be a $\leqq$-chain, and define $m \in H^{n}$ by 


$$
m_{i}=\inf \left\{x_{i}: x \in K\right\} \text {. }
$$

Then $m \leqq x$ for each $x \in K$, and since $K$ is a $\leqq$-chain, it is easy to see that for each $\varepsilon>0$ there is an $x \in K$ such that $m_{i} \leqq x_{i}<m_{i}+\varepsilon$ for $1 \leqq i \leqq n$. Consequently $m \in K^{-}$. It follows from the above lemma that $\leqq$ is a GLS-relation.

2.3. Proof of Theorem 4. Let $\leqq$ be a reflexive binary relation on $T$ such that $\{y \in T: x \leqq y\}$ is open in $T$ for each $x \in T$. Now $T$ is a Baire space as a subspace of $S$ (since it is a Baire space as a subspace of $R$ ). Hence one can easily find $\varepsilon>0$ and $a, b$ with $a<b$, and a dense subset $E$ of $(a, b) \cap T$, such that $[x, x+\varepsilon) \cap T \subseteq$ $\{y \in T: x \leqq y\}$ for each $x \in E$. Let $q$ be a rational number, $a<q<b$. There is a set $F=\left\{x_{n}: n \geqq 1\right\} \subseteq E$ such that

$$
q<x_{n+1}<x_{n}<q+\min \{\varepsilon, 1 / n\} \text { for } n \geqq 1 \text {. }
$$

Then $F$ is closed in $T$ but has no $\leqq$-minimal element, since $x_{n+1} \prec x_{n}$ (i.e., $x_{n+1} \leqq x_{n}$ and $x_{n+1} \neq x_{n}$ ) for all $n \geqq 1$.

3. Proof of Theorem 6 . Let $R$ be the real line with its usual topology. For $x \in R$ let $R_{x}=R \times\{x\}$.

3.1. The proof. For each $z \in R$ the set

$$
\nabla_{z}=\left\{\langle x, y\rangle \in S^{2}: x+y=z\right\}
$$

is closed and discrete in $S^{2}$. Hence it suffices to show that there are an $E \in \mathscr{E}$ and a $z \in R$ such that some separable, not necessarily closed, subspace intersects $\nabla_{z}$ in a set of cardinality $c$.

It is easy to see that if $A \subseteq R^{2}$ is dense in some open subspace of $R^{2}$, then $A$, considered as subspace of $S^{2}$, is separable. Since all sets $\nabla_{z}$ are lines of slope -1 , the theorem follows from the following lemma.

Lemma 3.2. Let $\mathscr{A}$ be any countable family of subsets of $R^{2}$ that covers $R^{2}$. Then there are an $A \in \mathscr{A}$ and a nonempty open $U$ in $R^{2}$ such that

$$
\cup\left\{R_{x} \cap A \cap U: x \in R,\left|R_{x} \cap A \cap U\right|=c\right\}
$$

is dense in $U$.

Proof. Let $\mathscr{B}$ be a countable base for $R^{2}$. We argue by contradiction, and suppose that for each $A \in \mathscr{A}$ and $B \in \mathscr{B}$ we can find a nonempty open $V(A, B) \subseteq B$ such that 


$$
\left|R_{x} \cap A \cap V(A, B)\right|<c \text { for all } x \in R .
$$

For $A \in \mathscr{A}$ let $W(A)=\cup\{V(A, B): B \in \mathscr{B}\}$. Then $W(A)$ is a dense open set in $R^{2}$, and since the union of countably many sets of cardinality less than $c$ has again cardinality less than $c$,

$$
\left|R_{x} \cap A \cap W(A)\right|<c \text { for all } x \in R .
$$

If $G=\cap\{W(A): A \in \mathscr{A}\}$, then $\left|R_{x} \cap A \cap G\right|<c$ for all $x \in R$. Since $G \subseteq \cup \mathscr{A}$, the same argument shows that

$$
\left|R_{x} \cap G\right|<c \text { for all } x \in R .
$$

However, this is impossible. For by [7], or [9, 15.1], every intersection of countably many dense open sets in $R^{2}$ intersects $R_{x}$ in a set that is dense in $R_{x}$ with respect to the subspace topology for at least one $x_{0}$ But then $R_{x} \cap G$ is a dense $G_{\delta}$ in $R_{x}$, hence has cardinality $c$, cf. $[9,5.1]$.

4. Appendix: GLS-spaces. GLS-spaces are useful in proving that certain spaces are $D$-spaces. Here we collect some of their basic properties. We omit straightforward proofs.

We did not postulate that a GLS-relation be transitive; an easy example shows that it need not be: define a GLS-relation $\leqq$ on the nonnegative integers by $k \leqq n$ iff $k \leqq n \leqq k+1$. (We did not attempt to decide whether or not every GLS-space admits a transitive GLS-relation.) On the other hand, since all spaces considered are $T_{1}$, we have the following.

Proposition 4.1. Every GLS-relation is antisymmetric.

This can be used to prove the following propositions.

Proposition 4.2. A compact Hausdorff space without isolated points is not a GLS-space.

The example of a countable space with the cofinite topology shows that the Hausdorff condition is essential. A collection $\mathscr{A}$ of subsets of a space $X$ is called a network for $X$ if for every open $U$ in $X$ and every $x \in U$ there is an $A \in \mathscr{A}$ with $x \in A \subseteq U$.

Proposition 4.3. If the space $X$ has a network $\mathscr{A}$ with $|\mathscr{A}|<|X|$, then $X$ is not a GLS-space. In particular, no uncountable separable metrizable space is a GLS-space.

The following proposition should be compared with analogous 
results for $D$-spaces [2].

Proposition 4.4. (a) A closed subspace of a GLS-space is a GLS-space.

(b) If $X=X_{1} \cup X_{2}$, with $X_{1}$ and $X_{2}$ GLS-spaces and $X_{1}$ closed, then $X$ is a GLS-space.

(c) A space is a GLS-space if it is the union of countably many closed subspaces, each of which is a GLS-space.

Note that it follows from 4.4 (a), and 4.2 or 4.3 that no infinite product of nontrivial spaces is a GLS-space. In particular, $S^{\omega}$ is not a GLS-space.

\section{REFERENCES}

1. E. K. van Douwen, Retracts of the Sorgenfrey line, Compos. Math., 38 (1979), $155-161$.

2. - Neighborhood assignments, in preparation.

3. The Pixley-Roy Topology on Spaces of Subsets, in "Set Theoretic Topology", ed. G. M. Reed, Academic Press, 1977, 111-134.

4. W. G. Fleissner, Separation axioms in Moore spaces, Fund, Math., 98 (1978), 279-286.

5. F. B. Jones, Concerning normal and completely normal spaces, Bull. Amer. Math. Soc., 43 (1937), 671-677.

6. I. Juhász, Cardinal functions in topology, Mathematical Centre Tract 34, Amsterdam, 1971.

7. K. Kuratowski and S. Ulam, Quelques propriétés topologiques du produit combinatoire, Fund. Math., 19 (1932), 247-251.

8. D. J. Lutzer, Another property of the Sorgenfrey line, Compos. Math., 24 (1972), 359-363.

9. J. C. Oxtoby, Measure and Category, Springer, Heidelberg, 1971.

Received January 4, 1977.

Institute For Medicine AND Mathematics

OHIO UNIVERSITY

ATHENS, OH 45701

AND

UNIVERSITY OF CALIForNiA

DAvIS, CA 95616 



\title{
PACIFIC JOURNAL OF MATHEMATICS
}

\section{EDITORS}

\author{
DONALD BABBITT (Managing Editor) \\ University of California \\ Los Angeles, CA 90024 \\ HUGo ROSSI \\ University of Utah \\ Salt Lake City, UT 84112 \\ C. C. MOORE \\ University of California \\ Berkeley, CA 94720
}

J. DugundJI

Department of Mathematics

University of Southern California

Los Angeles, CA 90007

R. FinN and J. Milgram

Stanford University

Stanford, CA 94305

\section{ASSOCIATE EDITORS}
E. F. BECKENBACH
B. H. Neumann
F. WolF
K. YOSHIDA

\section{SUPPORTING INSTITUTIONS}

\author{
UNIVERSITY OF BRITISH COLUMBIA \\ CALIFORNIA INSTITUTE OF TECHNOLOGY \\ UNIVERSITY OF CALIFORNIA \\ MONTANA STATE UNIVERSITY \\ UNIVERSITY OF NEVADA, RENO \\ NEW MEXICO STATE UNIVERSITY \\ OREGON STATE UNIVERSITY \\ UNIVERSITY OF OREGON
}

UNIVERSITY OF SOUTHERN CALIFORNIA

STANFORD UNIVERSITY

UNIVERSITY OF HAWAII

UNIVERSITY OF TOKYO

UNIVERSITY OF UTAH

WASHINGTON STATE UNIVERSITY

UNIVERSITY OF WASHINGTON

The Supporting Institutions listed above contribute to the cost of publication of this Journal, but they are not owners or publishers and have no responsibility for its content or policies.

Mathematical papers intended for publication in the Pacific Journal of Mathematics should be in typed form or offset-reproduced, (not dittoed), double spaced with large margins. Please do not use built up fractions in the text of the manuscript. However, you may use them in the displayed equations. Underline Greek letters in red, German in green, and script in blue. The first paragraph or two must be capable of being used separately as a synopsis of the entire paper. Please propose a heading for the odd numbered pages of less than 35 characters. Manuscripts, in triplicate, may be sent to any one of the editors. Please classify according to the scheme of Math. Reviews, Index to Vol. 39. Supply name and address of author to whom proofs should be sent. All other communications should be addressed to the managing editor, or Elaine Barth, University of California, Los Angeles, California, 90024.

50 reprints to each author are provided free for each article, only if page charges have been substantially paid. Additional copies may be obtained at cost in multiples of 50 .

The Pacific Journal of Mathematics is issued monthly as of January 1966. Regular subscription rate: $\$ 72.00$ a year (6 Vols., 12 issues). Special rate: $\$ 36.00$ a year to individual members of supporting institutions.

Subscriptions, orders for numbers issued in the last three calendar years, and changes of address should be sent to Pacific Journal of Mathematics, P.O. Box 969, Carmel Valley, CA 93924, U.S.A. Older back numbers obtainable from Kraus Periodicals Co., Route 100, Millwood, NY 10546.

PUBLISHED BY PACIFIC JOURNAL OF MATHEMATICS, A NON-PROFIT CORPORATION

Printed at Kokusai Bunken Insatsusha (International Academic Printing Co., Ltd.). 8-8, 3-chome, Takadanobaba, Shinjuku-ku, Tokyo 160, Japan.

Copyright (C) 1979 by Pacific Journal of Mathematics Manufactured and first issued in Japan 


\section{Pacific Journal of Mathematics}

\section{Vol. 81, No. $2 \quad$ December, 1979}

Ersan Akyildiz, Vector fields and equivariant bundles ............... 283

Ehrhard Behrends, The centralizer of tensor products of Banach spaces ( $a$ function space representation) ......................... 291

Geoffrey R. Burton, Congruent sections of a convex body ............. 303

John Warnock Carlson, $H$-closed and countably compact extensions ...... 317

Robert Charles Carlson, Eigenfunction expansions for selfadjoint

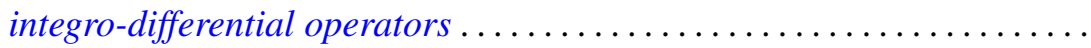

Robert Damiano, Coflat rings and modules.

Eric Karel van Douwen and Washek (Vaclav) Frantisek Pfeffer, Some properties of the Sorgenfrey line and related spaces ................

Uri Elias, Necessary conditions and sufficient conditions for disfocality and

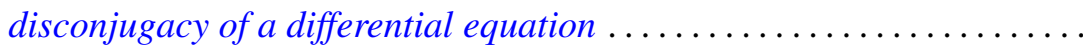

V. L. (Vagn Lundsgaard) Hansen, Polynomial covering spaces and homomorphisms into the braid groups .......................

Paul Hess, Dedekind's problem: monotone Boolean functions on the lattice

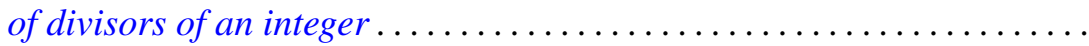

Alan Hopenwasser and David Royal Larson, The carrier space of a reflexive

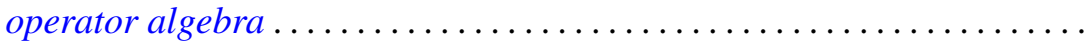

Kyung Bai Lee, Spaces in which compacta are uniformly regular $G_{\delta} \ldots \ldots$.

Claude Levesque, A class of fundamental units and some classes of

Jacobi-Perron algorithms in pure cubic fields...

Teck Cheong Lim, A constructive proof of the infinite version of the

Belluce-Kirk theorem ..........................

Dorothy Maharam and A. H. Stone, Borel boxes

Roger McCann, Asymptotically stable dynamical systems are linear

Peter A. McCoy, Approximation and harmonic continuation of axially symmetric potentials in $E^{3} \ldots \ldots \ldots \ldots \ldots \ldots \ldots$

Takahiko Nakazi, Extended weak-* Dirichlet algebras ....

Carl L. Prather, On the zeros of derivatives of balanced trigonometric polynomials ................................

Iain Raeburn, An implicit function theorem in Banach spaces...

Louis Jackson Ratliff, Jr., Two theorems on the prime divisors of zeros in completions of local domains...

Gloria Jean Tashjian, Cartesian-closed coreflective subcategories of

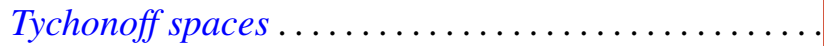

Stephen Edwin Wilson, Operators over regular maps.... . . 